\title{
Other top quark properties in CMS
}

\author{
Yvonne Kuessel ${ }^{* i}$ \\ RWTH Aachen university, Germany \\ E-mail: kuesselecern.ch
}

\begin{abstract}
Measurements of top-quark properties such as the first measurement of vector-boson production associated with top-quark pairs, the flavor contents in top-antitop events and constraints on the top-quark charge are presented. The analyses use proton-proton collision data recorded in 2011 with the CMS experiment at the LHC at a center-of-mass energy of $7 \mathrm{TeV}$.

A signal for associated vector boson production with top-quark pairs is established in the exclusive $t \bar{t} Z$ trilepton channel and in the inclusive $t \bar{t} V$ same-sign dilepton channel. The respective cross sections are determined.

The fraction of top quarks decaying into a $W$ boson and a $b$ quark relative to all top-quark decays, $R=B(t \rightarrow W b) / \sum(B(t \rightarrow W q))$, is determined in the dilepton final state by counting the jets identified as $b$ jets, using a model that relies on the $b$-tagging and mistagging efficiencies.

For the measurement of the top-quark charge, the top quark pair events are reconstructed in the muon+jets final state. Different top quark charge hypotheses $(+2 / 3$ e or $-4 / 3$ e $)$ are discriminated by using charge correlations between high- $p_{\mathrm{T}}$ muons from the hard process and muons from $B$ hadron decays in $b$ jets.
\end{abstract}

All results are consistent with SM predictions.

36th International Conference on High Energy Physics

4-11 July 2012

Melbourne, Australia

\footnotetext{
* Speaker.

${ }^{\dagger}$ On behalf of the CMS Collaboration
} 


\section{Introduction}

Being the heaviest elementary particle discovered so far, the top quark holds prominent properties making it a unique probe to test the standard model. At the LHC top-quark pair events are produced in large quantities enabling a detailed top quark characterization on a high-precision level. Though the top quark has been extensively studied since its discovery, some of its properties are still poorly known. The analyses presented in the following examine three of them.

The first analysis investigates the couplings to vector bosons. The associated production of topquark pairs with a vector boson is an important test of the standard model at the $\mathrm{TeV}$ scale and performed for the fist time with the CMS experiment [1].

The top quark decays in approx. $99.8 \%$ into a $W$ boson and a $b$ quark; a consequence of the unitarity of the CKM matrix and the other measured CKM elements. Deviations could arise from a fourth quark generation. A measurement [2] is presented which determines $R=B(t \rightarrow W b) / B(t \rightarrow W q)$ ( $B$ denotes the branching fraction and $q=b, s, d$ ) from the $b$-jet multiplicity of top-quark pairs in the dilepton-decay channel.

Being the electro-weak isospin partner of the bottom quark, the top quark is expected to carry an electric charge of $+2 / 3 \mathrm{e}$. A measurement [3] is presented that discriminates the SM expectation against an exotic top quark charge of $-4 / 3 \mathrm{e}$, a scenario compatible with charge conservation with respect to the decay products: a $W$ boson and a $b$ quark.

These analyses in the following presented in more detail use data recorded with the CMS experiment in 2011. A detailed description of the CMS detector can be found elsewhere [4].

\section{Vector-boson production associated with top-quark pairs}

The associated production of top-quark pairs with a vector boson is performed in two different channels using $4.98 \mathrm{fb}^{-1}$ of CMS data [1].

In the trilepton channel events are selected from the decay: $t \bar{t} Z \rightarrow(t \rightarrow b \ell v)(t \rightarrow b j j)(Z \rightarrow \ell \ell)$, exploiting the characteristic signature of the final state by requiring two opposite-charge, sameflavor leptons (electrons, muons) considered as $Z$ candidate. A third lepton and at least three jets (including two $b$ jets) must be in the event. SM backgrounds with the same final state are negligible but wrongly identified leptons and $b$ jets constitute other backgrounds significantly, mainly $t \bar{t}$, $Z+$ jets and diboson (e.g. $W Z$ ) processes. A scale factor of 1.05 for the $t \bar{t}$ contribution is extracted from data by fitting the invariant mass distribution from cross-flavor pairs passing a preselection (loosened two lepton criteria, three jets). The scale factor of 1.32 for the $Z+j e t s$ and diboson MC yields is estimated with the invariant mass distribution of the $Z$ candidate on the preselected events fulfilling additionally the third lepton requirement and a veto on events with $b$ jets.

The dilepton analysis selection, as well optimized for signal significance, searches events from the decays $t \bar{t} W \rightarrow\left(t \rightarrow b \ell^{ \pm} v\right)(t \rightarrow b j j)\left(W \rightarrow \ell^{ \pm} v\right)$ and $t \bar{t} Z \rightarrow\left(t \rightarrow b \ell^{ \pm} v\right)(t \rightarrow b j j)\left(Z \rightarrow \ell^{ \pm} \ell^{\mp}\right)$ by requiring two isolated, same-charge leptons, three jets (including one $b$ jet). There is a veto on the trilepton selection to guarantee statistical independence. Same-sign dilepton processes are rare in the SM. Many of the backgrounds result from mis-reconstruction. They can be estimated from control regions in data. Defining a loose and tight isolation/identification, the ratio of loose leptons passing the tight criteria is measured. $Z$ candidate events are used for real leptons. Fake 

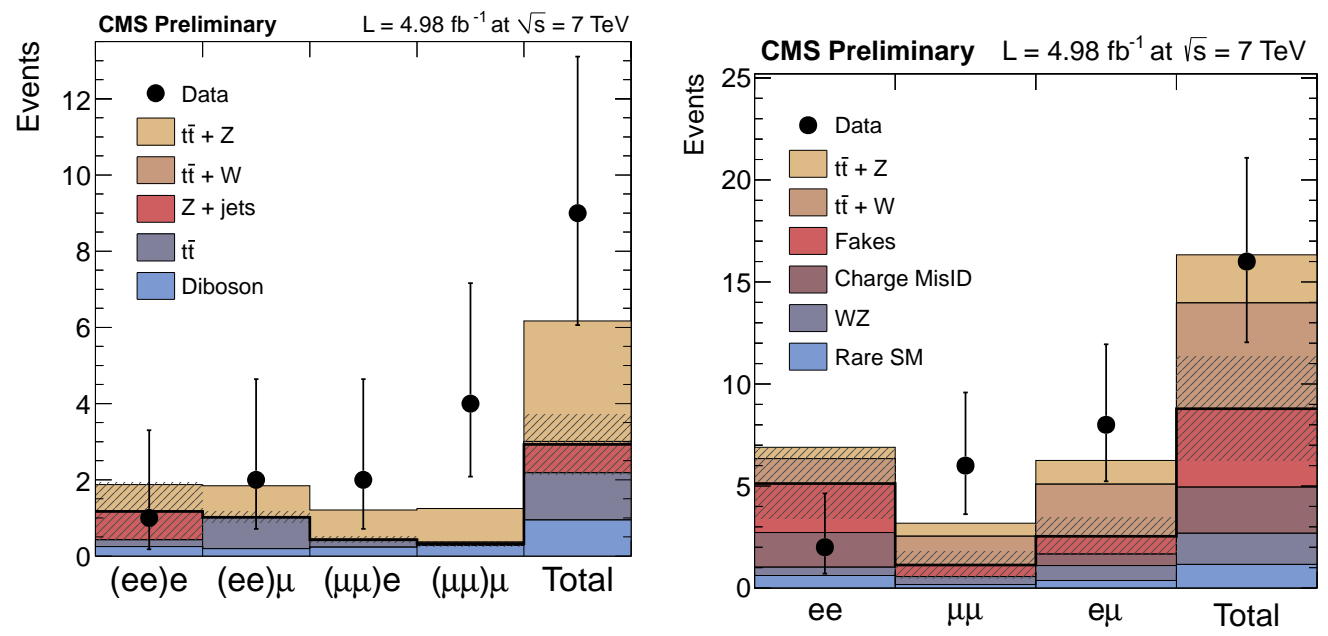

Figure 1: Event yields in the various trilepton (left) and dilepton (right) channels after the final selection requirements. The gray diagonal hashed band corresponds to the uncertainty on the background yield. [1]

or non-prompt lepton ratios are determined from a QCD-dominated control sample with no significant missing transverse energy. The charge mis-identification rate for electrons is measured to be $0.02 \%(0.3 \%)$ in the barrel (endcap) from the fraction of same-signed electrons in $Z$ decays. For the $W Z$ cross section the CMS measurement [5] is used. Remaining rare SM processes (e.g. di/triboson events) are taken from MC prediction.

The exclusive results for the trilepton and dilepton channel with a significance of 3.66 and 2.99 standard deviations, respectively, are listed in Table 1 and shown in Figure 1. The trilepton cross section is translated into an inclusive $t \bar{t} V$ cross section by taking into account the $t \bar{t} V$ selection efficiency of $\varepsilon=0.21 \%$ determined from the simulation. Additionally, the $t \bar{t} W$ cross section can be extracted from the dilepton result with 2.44 standard deviations by subtracting the expected $t \bar{t} Z$ yield from simulation. By multiplying all seven decay channels the combined $t \bar{t} V$ cross section is measured with a significance of 4.67 standard deviations above the background hypothesis. All systematic uncertainties are treated as uncorrelated except for the uncertainties of the backgrounds. The result is larger compared to the NLO calculation but still compatible.

Table 1: Measured cross sections of the $t \bar{t} Z$ [6], $t \bar{t} W$ [7] and $t \bar{t} V$ processes in the tri-, dilepton and combined channels compared to NLO predictions. The first of the uncertainties on the measurements refers to the statistical, the second to the systematic component. No uncertainty is available for the $\sigma(t \bar{t} Z)$ prediction.

\begin{tabular}{rccc}
\hline \hline & $\sigma(t \bar{t} Z)[\mathrm{pb}]$ & $\sigma(t \bar{t} W)[\mathrm{pb}]$ & $\sigma(t \bar{t} V)[\mathrm{pb}]$ \\
\hline Trilepton Analysis & $\mathbf{0 . 3 0}{ }_{-\mathbf{0 . 1 1}}^{+\mathbf{0 . 1 4}+\mathbf{0 . 0 2}}$ & - & $0.66_{-0.25}^{+0.32}+0.09$ \\
Dilepton Analysis & - & $0.28_{-0.12}^{+0.14} \pm 0.04$ & $\mathbf{0 . 4 5}{ }_{-\mathbf{0 . 1 5}}^{+0.15}+\mathbf{0 . 0 5}$ \\
Combined & - & - & $0.51_{-0.13}^{+0.05}{ }_{-0.04}^{+0.05}$ \\
NLO Calculation & 0.1387 & $0.169_{-0.051}^{+0.029}$ & 0.308 \\
\hline \hline
\end{tabular}




\section{Measuring the heavy-flavor content in top-pair events}

The measurement of $R=B(t \rightarrow W b) / B(t \rightarrow W q)$ in the dilepton-decay channel uses $2.2 \mathrm{fb}^{-1}$ of data [2]. Additionally to double-lepton triggers the selection requires at least two isolated high$p_{\mathrm{T}}$ leptons (electron, muon). If more than two leptons are selected, the opposite-charge and highest sum of transverse momenta pair is selected. Same-flavor pairs are rejected if they are compatible with $Z \rightarrow \ell \ell$ decays. Quarkonium decays are suppressed by a requirement of $M_{\ell \ell}>12 \mathrm{GeV}$. Two jets are required in the event that are well separated from the selected leptons. $E_{T}^{\text {miss }}>30 \mathrm{GeV}$ for final states with same-flavor leptons suppresses DY processes, the largest background in this channel. Its yield is estimated from data by fitting the distribution of the angle between the leptons exploiting the angular correlations occurring in DY events in contrast to top-pair events. The template for DY is taken from the low $E_{T}^{\text {miss }}<30 \mathrm{GeV}$ region. The fraction of $t \bar{t}$ events in the selected sample is $f_{t \bar{t}}=0.767 \pm 0.052$ (stat+syst).

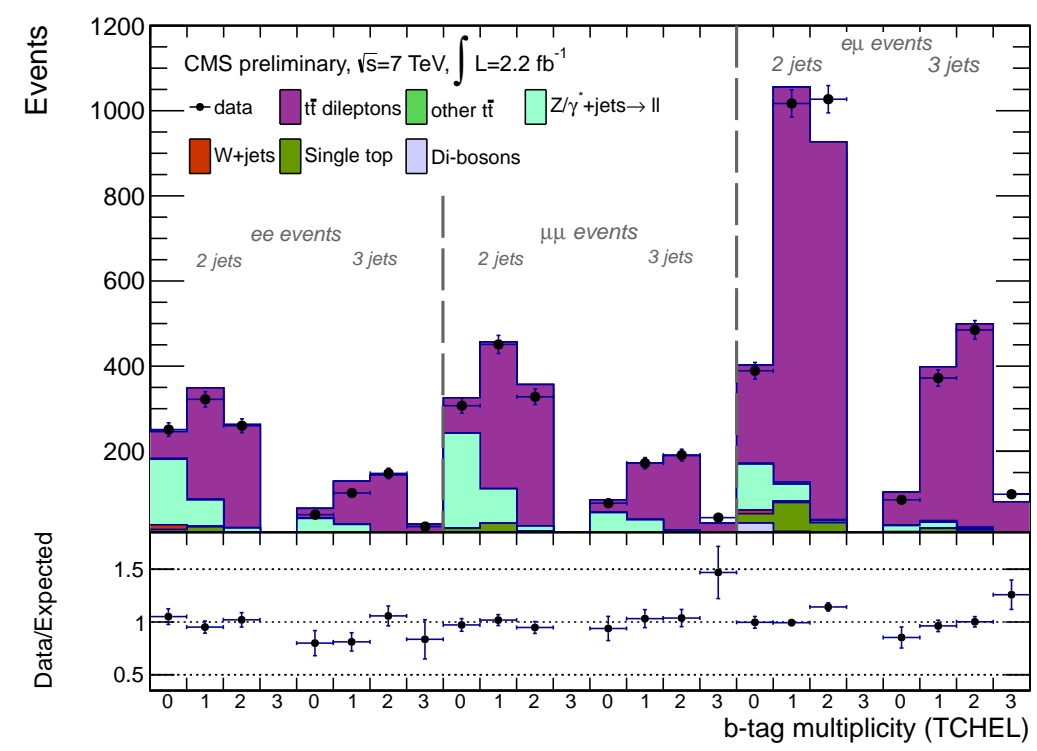

Figure 2: Number of $b$-tagged jets per event in the different dilepton channels for events with two or three jets respectively. The simulation corresponds to $R=1$. [2]

The $b$ jets are identified with the Track Counting High Efficiency algorithm [8] of which the efficiency is measured from data using dijet QCD events. The residual differences in Figure 2 result from the number of selected jets from top-quark decays or from ISR. This is determined from data by fitting the lepton-jet invariant mass spectrum shown in Figure 3 (left) with correct assignments restricted by $M_{l, j} \approx \sqrt{m_{t}^{2}-m_{W}^{2}}=156 \mathrm{GeV}$. A data-driven model for the mis-assignment contribution is used. The $b$-jet multiplicity can be modeled as a function of $R, b$-tagging efficiencies and the contribution of jets from top or other sources. The result of a binned likelihood fit using 21 event categories with two or three jets and different dilepton channels is shown in Figure 3 (right).

The measured value for $R=0.98 \pm 0.04$ is dominated by uncertainties on the $b$-tagging efficiency $(\approx 3 \%)$ and the fraction of correct assignments $(\approx 2 \%)$. The statistical uncertainty is 

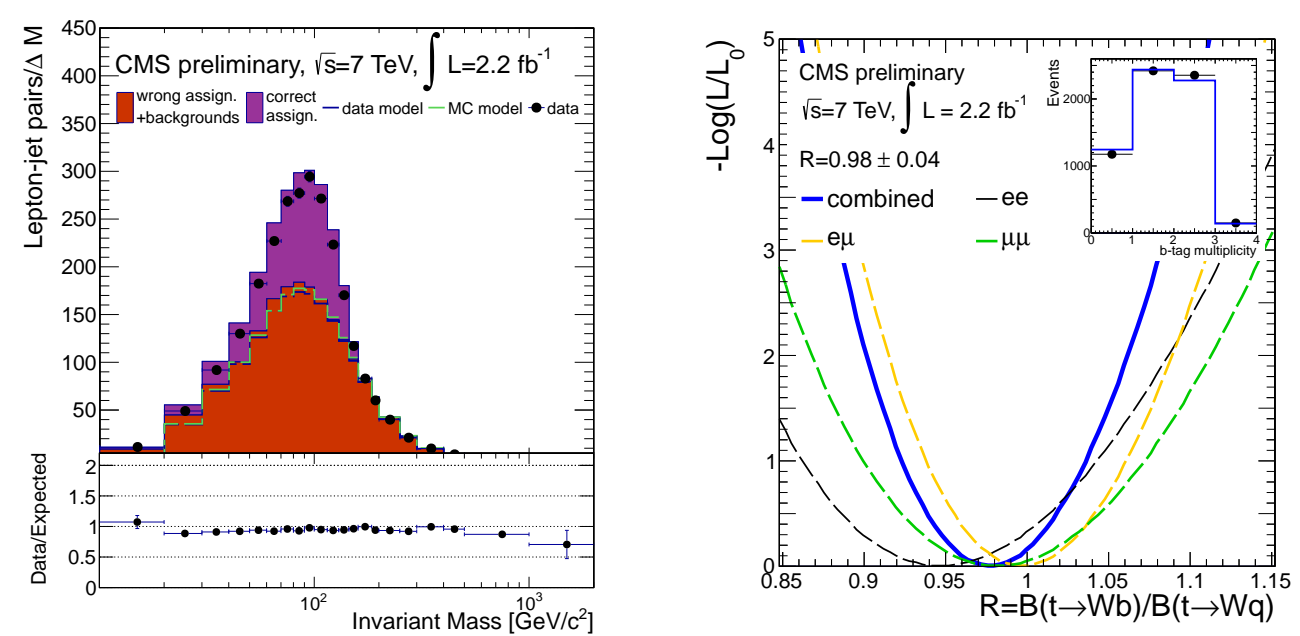

Figure 3: Left: Invariant mass of the lepton-jet pairs for all possible lepton-jet assignments. Using MC truth, the assignments are classified into correct and wrong jet-assignments. Right: Variation of the profile likelihood for the individual dilepton channels and all channels combined. The inset shows the inclusive $b$-jet multiplicity and the final fit. [2]

about $2 \%$. A $95 \%$ C.L. interval for $R$ as $0.85-1.0$ is derived taking into account the statistical and systematic uncertainties.

\section{Constraints on the top quark electric charge}

For the measurement of the top-quark charge [3], the top quark pair events are reconstructed in the muon+jets final state. Having applied a standard top quark pair selection, the $W$-boson charge can be determined from the high- $p_{\mathrm{T}}$ muon of the hard process. The charge sign of the $b$ quark can be tagged by a muon occurring in $B$ meson decays within the $b$ jet. The muon is required to have a $p_{\mathrm{T}}>4 \mathrm{GeV}$. The probability of correct charge assignment $P_{\mathrm{bC}}$ increases with rising momentum relative to the jetaxis $\left(p_{\mathrm{T}}^{\text {rel }}\right)$ due to a suppression of muons from lighter $D$ mesons that have opposite charges compared to the initial $b$ quarks. Requiring $p_{\mathrm{T}}^{\text {rel }}>0.85 \mathrm{GeV}$, a correct charge tagging in $P_{\mathrm{bC}}=[74.5 \pm 0.8$ (stat.) \pm 2.6 (syst.) $] \%$ of all cases is reached. This is validated on an independent dijet data sample.

Exploiting the top mass on the hadronic decay side the charge-tagged $b$ jet is assigned to the leptonic or hadronic decay side. A quality cut on the invariant mass of the leptonic $b$ jet and the high- $p_{\mathrm{T}}$ muon and on the hadronic top candidate mass results in a correct assignment of the $b$ jet in $P_{\text {pair }}=[80.2 \pm 0.8$ (stat.) \pm 3.8 (syst.) $] \%$ of all cases.

Combining the charges of the $W$ boson and the $b$ jet a charge hypothesis of either $-2 / 3 \mathrm{e}$ or $+4 / 3 \mathrm{e}$ can be build, shown in Figure 4 (left). The data agrees with the SM simulation. This is interpreted in statistical terms using the normalized asymmetry $A$ between the two bins as test statistics. Pseudo experiments are generated with and without systematic uncertainties leading to the probability density function of $A$. The measurement of $A_{\text {meas }}=0.97 \pm 0.12$ (stat.) \pm 0.31 (sys.) is limited by systematic uncertainties. The procedure for discriminating the two top-quark charge hypotheses is based on the determination of the probability to reject the exotic hypothesis assuming 
it is correct (called "error of type I" and related to a parameter $\alpha$ ) and the probability to reject the standard model hypothesis assuming it is correct (called "error of type II" and related to a parameter $\beta$ ). The result is shown in Figure 4 (right). For the hypothesis test, the type I error rate is chosen a priori where the type I and type II error rates are equal. This corresponds to $\alpha=0.001$ resulting in a $99.9 \%$ CL exclusion confirming nicely the SM expectation.
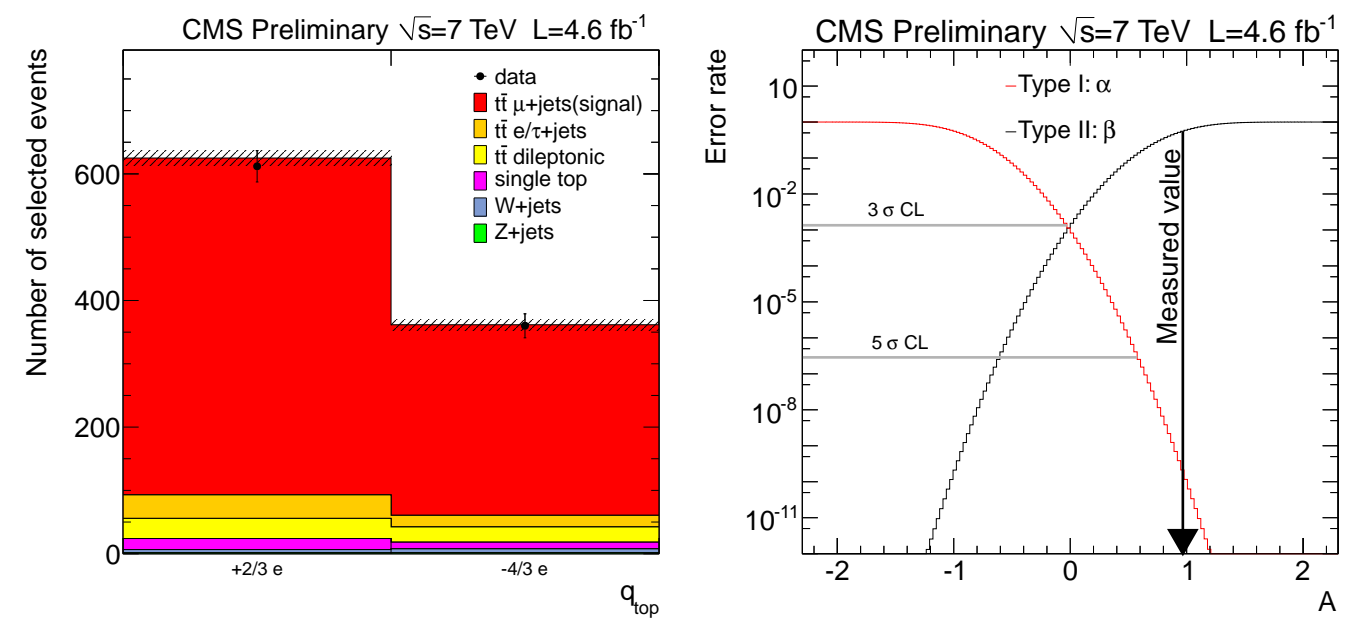

Figure 4: Left: Charge assigned to the top quark in the selected top-pair events. The data are compared to the standard-model simulation. Right: Type I and II error rates for the hypothesis test, including all systematic uncertainties. [3]

\section{References}

[1] the CMS Collaboration. Measurement of the Associated Production of Vector Bosons with Top-Antitop Pairs at 7 TeV. CMS Physics Analysis Summary, (2012). CMS-PAS-TOP-12-014.

[2] the CMS Collaboration. First measurement of $B(t \rightarrow W b) / B(t \rightarrow W q)$ in the dilepton channel in pp collisions at $\sqrt{s}=7$ TeV. CMS Physics Analysis Summary, (2012). CMS-PAS-TOP-11-029.

[3] the CMS Collaboration. Constraints on the Top-Quark Charge from Top-Pair Events. CMS Physics Analysis Summary, (2012). CMS-PAS-TOP-11-031.

[4] the CMS Collaboration. The CMS experiment at the CERN LHC. JINST 0803, (2008) S08004.

[5] the CMS Collaboration. Measurement of the WW, WZ and ZZ cross sections at CMS. CMS Physics Analysis Summary, (2012). CMS-PAS-EWK-11-010.

[6] J. M. Campbell, R. K. Ellis. $t \bar{t} W$ production and decay at NLO. FERMILAB-PUB-12-109-T, (2012). arXiv:1204.5678.

[7] A. KARdos, Z. TROCSANyi, AND C. PAPAdOPOUlOS. Z0 - boson production in association with a top anti-top pair at NLO accuracy with parton shower effects. Phys. Rev. D 85, 074022, (2012). doi:10.1103/PhysRevD.85.074022, arXiv:1111.1444.

[8] The CMS Collaboration. b-Jet Identification in the CMS Experiment. CMS Physics Analysis Summary, (2012). CMS PAS BTV-11-004 